TRANSACTIONS OF THE

AMERICAN MATHEMATICAL SOCIETY

Volume 356, Number 1, Pages 61-71

S 0002-9947(03)03388-9

Article electronically published on August 25, 2003

\title{
SEMI-LINEAR HOMOLOGY $G$-SPHERES AND THEIR EQUIVARIANT INERTIA GROUPS
}

\author{
ZHI LÜ
}

\begin{abstract}
This paper introduces an abelian group $H \Theta_{V}^{G}$ for all semi-linear homology $G$-spheres, which corresponds to a known abelian group $\Theta_{V}^{G}$ for all semi-linear homotopy $G$-spheres, where $G$ is a compact Lie group and $V$ is a $G$-representation with $\operatorname{dim} V^{G}>0$. Then using equivariant surgery techniques, we study the relation between both $H \Theta_{V}^{G}$ and $\Theta_{V}^{G}$ when $G$ is finite. The main result is that under the conditions that $G$-action is semi-free and $\operatorname{dim} V-\operatorname{dim} V^{G} \geq 3$ with $\operatorname{dim} V^{G}>0$, the homomorphism $T: \Theta_{V}^{G} \longrightarrow H \Theta_{V}^{G}$ defined by $T\left([\Sigma]_{G}\right)=\langle\Sigma\rangle_{G}$ is an isomorphism if $\operatorname{dim} V^{G} \neq 3,4$, and a monomorphism if $\operatorname{dim} V^{G}=4$. This is an equivariant analog of a well-known result in differential topology. Such a result is also applied to the equivariant inertia groups of semi-linear homology $G$-spheres.
\end{abstract}

\section{INTRODUCTION}

Let $\Theta_{n}$ (resp. $H \Theta_{n}$ ) be the set of consisting of the $h$-cobordism (resp. homology cobordism) classes of all homotopy (resp. homology) spheres. It is well known that both $\Theta_{n}$ and $H \Theta_{n}$ form abelian groups under connected sum operation, and for $n \neq 3, \Theta_{n}$ is finite (see $[\mathrm{KM}]$ ). Note that the group structure of $\Theta_{3}$ is still open (this is associated to Poincaré conjecture in dimension three), but $\mathrm{H}_{3}$ must be infinite as noticed by Fintushel and Stern [FS] (see also [Fu]). With respect to the relation between $\Theta_{n}$ and $H \Theta_{n}$, there is a natural homomorphism $T_{n}: \Theta_{n} \longrightarrow H \Theta_{n}$ defined by $T_{n}\left(\left[\Sigma^{n}\right]\right)=\left\langle\Sigma^{n}\right\rangle$ for $\left[\Sigma^{n}\right] \in \Theta_{n}$. Then the following result follows from $\mathrm{HH}]$ and [Ke.

Theorem 1.1. The homomorphism $T_{n}: \Theta_{n} \longrightarrow H \Theta_{n}$ is an isomorphism for $n \neq 3$.

As a consequence of Theorem 1.1, one has

Corollary 1.2. For any $\left\langle M^{n}\right\rangle \in H \Theta_{n}$ with $n \neq 3$, then the inertia group $I\left(M^{n}\right)$ of $M^{n}$ is trivial, i.e., $I\left(M^{n}\right)=(0)$.

Note that the inertia group $I\left(M^{n}\right)$ of a closed oriented manifold $M^{n}$ is defined as the group of those homotopy spheres $\Sigma^{n}$ satisfying the condition that the connected

Received by the editors July 3, 2000.

2000 Mathematics Subject Classification. Primary 57S15, 57S17, 57R91, 57R55, 57R67.

$K e y$ words and phrases. Semi-linear homology $G$-sphere, equivariant inertia group, $G$-action, representation, surgery.

This work was supported by the Japanese Government Scholarship, and partially supported by the research fund of the Ministry of Education in China and the JSPS Postdoctoral Fellowship (No. P02299). 
sum $M^{n} \sharp \Sigma^{n}$ is orientation preserving diffeomorphic to $M^{n}$. Such a group $I\left(M^{n}\right)$ may be used to classify the distinct differentiable structures almost diffeomorphic to $M^{n}$, or in other words, it may be used to measure the nonuniqueness of connected sums of $M^{n}$ with exotic spheres. The inertia groups for some special manifolds have been studied (see, for example [Ko, [Sc1, [Wi1], Wi2], Win]).

The purpose of this paper is to discuss the equivariant analogs of the above results. There are mainly three kinds of equivariant analogs, $\Theta_{V}^{G}, \Theta_{V}^{G, s}$, and $\Gamma_{V}^{G}$, of $\Theta_{n}$, where $G$ is a compact Lie group and $V$ is a representation of $G$. These three equivariant analogs are isomorphic to each other in most cases, and are often infinite (see [MSc1], MSc2]). Here we are only concerned with $\Theta_{V}^{G}$ (where $\Theta_{V}^{G}$, which consists of $G$-h-cobordism classes of all semi-linear homotopy $G$-spheres such that the tangential spaces at fixed points are equivalent to $V$, forms an abelian group under the equivariant connected sum if $\operatorname{dim} V^{G}>0$ ). In contrast to $\Theta_{V}^{G}$, we will construct an equivariant analog $H \Theta_{V}^{G}$ of $H \Theta_{n}$. Then we will study the relation between $\Theta_{V}^{G}$ and $H \Theta_{V}^{G}$ when $G$ is finite. It should be pointed out that it is possible to work very successfully with the other two groups $\Theta_{V}^{G, s}$ and $\Gamma_{V}^{G}$ and to get corresponding comparative results. The argument would require some additional input involving Rothenberg's work on classifying semi-linear actions on disks by equivariant Whitehead torsion, but this will be beyond what one wants to consider at this point.

$\Theta_{V}^{G}$ works in homotopy sense, while $H \Theta_{V}^{G}$ will work in homology sense. Hence, in order to construct $H \Theta_{V}^{G}$, we need to give the definitions of semi-linear homology $G$-spheres, $G$-equivariant homology cobordism and $G$-homology equivalence. These notations correspond to those known notations of semi-linear homotopy $G$-spheres, $G$ - $h$-cobordism and $G$-homotopy equivalence, respectively. Then $H \Theta_{V}^{G}$ is defined as the set of $G$-equivariant homology cobordism classes of all semi-linear homology $G$-spheres whose tangential spaces at fixed points are equivalent to $V$. We shall see that $H \Theta_{V}^{G}$ also forms an abelian group under the equivariant connected sum if $\operatorname{dim} V^{G}>0$. Furthermore, by making use of equivariant surgery techniques, the relation between both $\Theta_{V}^{G}$ and $H \Theta_{V}^{G}$ is discussed under the assumption that $G$ is finite and $G$-action is semi-free (i.e., $G$-action with two orbit types-fixed points and free orbits). The result is stated as follows.

Theorem 1.3. Suppose $G$ is a finite Lie group, and $V$ is a semi-free $G$-representation with $\operatorname{dim} V-\operatorname{dim} V^{G} \geq 3$ and $\operatorname{dim} V^{G}>0$. Then the homomorphism

$$
T: \Theta_{V}^{G} \longrightarrow H \Theta_{V}^{G}
$$

defined by $T\left([\Sigma]_{G}\right)=\langle\Sigma\rangle_{G}$ is an isomorphism if $\operatorname{dim} V^{G} \neq 3,4$, and a monomorphism if $\operatorname{dim} V^{G}=4$.

Remark. It is possible that the discussions for the relation between $\Theta_{V}^{G}$ and $H \Theta_{V}^{G}$ may be carried out if $G$-actions are not semi-free. Such an example may be provided when $G=\mathbb{Z}_{p^{r}}$ for $p$ a prime and $r \geq 2$ and if $G$-action is assumed to be not semi-free. Since the subgroups of $\mathbb{Z}_{p^{r}}$ are always linearly ordered and the isotropy subgroups

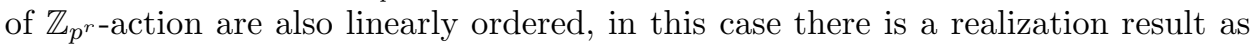
in Theorem 1.3. However, it seems difficult to determine to what extent Theorem 1.3 extends to non-semi-free actions.

The equivariant inertia group of a closed, equivariantly oriented smooth $G$ manifold has been introduced and studied by Masuda and Schultz; for details, 
see [Ma, MSc1, MSc2]. Applying Theorem 1.3 to the equivariant inertia group immediately gives the following result.

Corollary 1.4. Suppose $G$ is a finite Lie group and $V$ is a semi-free $G$-representation with $\operatorname{dim} V-\operatorname{dim} V^{G} \geq 3$ and $\operatorname{dim} V^{G}>0$. For $\langle M\rangle_{G} \in H \Theta_{V}^{G}$, the equivariant inertia group $I^{G}\left(M ; M^{G}\right)$ is trivial if $\operatorname{dim} V^{G} \neq 3$.

The arrangement of this paper is as follows. In Section 2 an equivariant ana$\log H \Theta_{V}^{G}$ of $H \Theta_{n}$ is constructed for $G$ to be a compact Lie group. In Section 3 we discuss the relationship between both $H \Theta_{V}^{G}$ and $\Theta_{V}^{G}$ when $G$ is finite, and give the proof of Theorem 1.3. The methods that we use are mainly equivariant spherical modification and equivariant handle addition. Throughout this paper, all $G$-manifolds, with or without boundary, are to be compact, smooth and $G$-oriented. The $G$-manifold $M$ with $G$-orientation reversed is denoted by $-M$.

\section{Construction of $H \Theta_{V}^{G}$}

Throughout this section, suppose that $G$ is a compact Lie group (here $G$ is not necessarily restricted to be finite) and $V$ is a $G$-representation. The task of this section is to construct an analog $H \Theta_{V}^{G}$ of $H \Theta_{n}$. As stated in Section 1, we wish that $H \Theta_{V}^{G}$ is comparable with $\Theta_{V}^{G}$. For this, we first review some standard notations with respect to $\Theta_{V}^{G}$.

Recall from Sc2 and [Sc3] that a semi-linear homotopy $G$-sphere is a manifold for which (1) the fixed point set of all subgroups are homotopy spheres, (2) if the fixed point set of one subgroup is a codimension 2 submanifold of the fixed point set of another subgroup, then the former is homotopically unknotted in the latter. An equivariant $h$-cobordism between such objects is an $h$-cobordism with group action such that the fixed point sets of all subgroups are $h$-cobordisms and there is an analog of the homotopy unknotting condition. Then $\Theta_{V}^{G}$ is defined as the set of the $G$ - $h$-cobordism classes of all those $G$-manifolds that are $G$-homotopically equivalent to $S(V \oplus \mathbb{R})$ and whose tangent spaces at fixed points are equivalent to $V$, where the $G$-action on $\mathbb{R}$ is trivial and $S(V \oplus \mathbb{R})$ is a linear $G$-sphere. $\Theta_{V}^{G}$ forms an abelian group under the equivariant connected sum operation if $\operatorname{dim} V^{G}>0$.

Based on the above, the construction of $H \Theta_{V}^{G}$ needs the following notations. Remember that $H \Theta_{V}^{G}$ should work in homology sense.

Definition 2.1. A homology $G$-sphere $M$ is a semi-linear homology $G$-sphere if $M^{H}$ is also a homology sphere for each subgroup $H$ of $G$. Note that Alexander duality ensures that a semi-linear homology $G$-sphere $M$ always possesses the homology unknottedness, i.e., for $H \leq L \leq G$ and $\operatorname{dim} M^{H}-\operatorname{dim} M^{L} \geq 1, M^{H}-M^{L}$ is homology equivalent to $S^{l}$ where $l=\operatorname{dim} M^{H}-\operatorname{dim} M^{L}-1$.

Clearly, a semi-linear homotopy $G$-sphere is a semi-linear homology $G$-sphere. For a semi-linear homology $G$-sphere $M$ on which $G$ acts semi-freely, since $M^{G}$ is also a homology sphere, $M^{G}$ is either connected (when $\operatorname{dim} M^{G}>0$ ) or $S^{0}$ (when $\operatorname{dim} M^{G}=0$ ). Then by $\left[\mathrm{AB}\right.$, [Mi2], the tangent space at each point $x \in M^{G}$ is equivalent to $V^{\prime}$, where $V^{\prime}$ is a given semi-free $G$-representation.

Definition 2.2. Two closed oriented smooth $G$-manifolds $M_{1}, M_{2}$ are $G$-equivariantly homologically cobordant if there exists an oriented compact smooth $G$-manifold $W$ such that the boundary $\partial W^{H}=M_{1}^{H} \sqcup-M_{2}^{H}$ and the inclusions $i_{j}: M_{j} \longrightarrow$ 
$W, j=1,2$, induce isomorphisms $H_{*}\left(M_{j}^{H} ; \mathbb{Z}\right) \cong H_{*}\left(W^{H} ; \mathbb{Z}\right)(j=1,2)$ for each subgroup $H$ of $G$.

It is obvious that $G$-equivariant homology cobordism is an equivalence relation, and for two oriented $G$-manifolds $M_{1}, M_{2}$, if $M_{1}$ and $M_{2}$ are $G$-equivariantly $h$ cobordant, then they are $G$-equivariantly homologically cobordant. In homotopy category, it is well known that a $G$-map $f: X \longrightarrow Y$ is a $G$-homotopy equivalence if and only if $f^{H}: X^{H} \longrightarrow Y^{H}$ is an ordinary homotopy equivalence for each subgroup $H$ of $G$. However, in homology category there does not exist the above analogous result in general. We say that a $G$-map $f: X \longrightarrow Y$ is a $G$-homology equivalence (or both $G$-spaces $X$ and $Y$ are $G$-homologically equivalent) if $f$ induces an isomorphism $H_{*}\left(X^{H} ; \mathbb{Z}\right) \cong H_{*}\left(Y^{H} ; \mathbb{Z}\right)$ for each subgroup $H$ of $G$.

With the above understood, we define $H \Theta_{V}^{G}$ as the set of the $G$-equivariant homology cobordism classes of all those $G$-manifolds that are $G$-homologically equivalent to $S(V \oplus \mathbb{R})$ and whose tangent spaces at fixed points are equivalent to $V$. $H \Theta_{V}^{G}$ can form an abelian group with respect to the equivariant connected sum if $\operatorname{dim} V^{G}>0$. In fact, it is easy to see that the equivariant connected sum of two semi-linear homology $G$-spheres is also a semi-linear homology $G$-sphere, and the equivariant connected sum operation is associative and commutative up to equivariantly orientation preserving differmorphism. The sphere $S(V \oplus \mathbb{R})$ serves as the identity element. Note that the condition $\operatorname{dim} V^{G}>0$ makes sure that the equivariant connected sum is commutative in $H \Theta_{V}^{G}$. It remains to check that each element of $H \Theta_{V}^{G}$ has an inverse.

Definition 2.3. A compact $G$-manifold $W$ is said to be equivariantly acyclic if $W^{H}$ is acyclic for any subgroup $H$ of $G$.

In nonequivariant topology, there are two well-known results with respect to $h$ cobordism, contractible manifolds and homotopy spheres: (1) a simply connected manifold $M^{n}$ is $h$-cobordant to the sphere $S^{n}$ if and only if $M^{n}$ bounds a contractible manifold; (2) if $M^{n}$ is a homotopy sphere, then $M^{n} \sharp\left(-M^{n}\right)$ bounds a contractible manifold (see [KM, Lemmas 2.3 and 2.4]). Those methods used to prove the above two results can be carried out to obtain the analogous results in homology sense, and especially can still work in equivariant cases. The proofs of the following results are straightforward. We would like to leave them as an exercise to the reader.

Lemma 2.1. Suppose $M$ is a closed oriented $G$-manifold such that the tangential spaces at all points of $M^{G}$ are equivalent to $V$. Then $M$ equivariantly bounds an equivariantly acyclic $G$-manifold $W$ if and only if $M$ is $G$-equivariantly homologically cobordant to $S(V \oplus \mathbb{R})$, i.e., $M$ is a representative of the identity element of $H \Theta_{V}^{G}$.

Lemma 2.2. If $M$ is a semi-linear homology $G$-sphere, then $M \sharp(-M)$ equivariantly bounds an equivariant acyclic $G$-manifold.

Now let $\langle M\rangle_{G}$ be an element in $H \Theta_{V}^{G}$. By Lemmas 2.1 and $2.2, M \sharp(-M)$ is $G$ equivariantly homologically cobordant to $S(V \oplus \mathbb{R})$, and thus $\langle-M\rangle_{G}$ is the inverse element of $\langle M\rangle_{G}$.

Combining the above arguments, we have

Proposition 2.2. If $\operatorname{dim} V^{G}>0$, then $H \Theta_{V}^{G}$ forms an abelian group under the equivariant connected sum operation. 


\section{Relation Between $H \Theta_{V}^{G}$ AND $\Theta_{V}^{G}$}

This section is devoted to complete the proof of Theorem 1.3. The proof relies on the following two lemmas.

Lemma 3.1. Let $G$ be a finite Lie group, and let $V$ be a semi-free $n$-dimensional $G$-representation such that $\operatorname{dim} V-\operatorname{dim} V^{G} \geq 3$ and $\operatorname{dim} V^{G}=k \geq 5$. For any $\langle M\rangle_{G} \in H \Theta_{V}^{G}$, there exists a semi-linear homotopy $G$-sphere $\Sigma$ that is $G$ equivariantly homologically cobordant to $M$.

Lemma 3.2. Let $G$ be a finite Lie group, and let $V$ be a semi-free $n$-dimensional $G$-representation such that $\operatorname{dim} V-\operatorname{dim} V^{G} \geq 3$ and $\operatorname{dim} V^{G}=k \geq 4$. Let $\langle M\rangle_{G} \in$ $H \Theta_{V}^{G}$; if $\langle M\rangle_{G}=0$, i.e., $M$ bounds an equivariantly acyclic $G$-manifold $W$, then it bounds an equivariantly contractible $G$-manifold.

We shall use equivariant spherical modification and equivariant handle addition to prove Lemmas 3.1 and 3.2. In our case, since $G$-actions are assumed to be semifree, i.e., the isotropy subgroups of $G$ only are $\{1\}$ and itself $G$, the equivariant surgery on $M$ in Lemma 3.1 (resp. $W$ in Lemma 3.2) is divided into two stages. The first one is to do surgery on the fixed point set $M^{G}$ (resp. $W^{G}$ ) and then on the $G$ free part $M-M^{G}$ (resp. $\left.W-W^{G}\right)$. Fortunately we have not encountered any surgery obstruction because all Stiefel-Whitney classes of $M^{H}$ (resp. $W^{H}$ ) are trivial for $H=\{1\}, G$, and in particular, only 1-dimensional and 2-dimensional surgeries are needed. Note that the triviality of the normal bundle of imbedded 1-dimensional (resp. 2-dimensional) sphere in ambient manifold is detected by the first (resp. second) Stiefel-Whitney class of ambient manifold. Also, according to [KM], when doing 1-dimensional surgery on a spin manifold, the resulting manifold may still preserve the spin structure. These facts will not be mentioned again in the proofs of Lemmas 3.1 and 3.2.

Proof of Lemma 3.1. Without loss of generality we assume that $M^{H}$ is not simply connected for $H=\{1\}, G$. Note that by $\left[\mathrm{Ke}\right.$, the fundamental group of $M^{H}$ has a finite presentation. Let $\Gamma=M \times I$, where $I$ is the unit interval $[0,1]$ with trivial $G$-action, and let $M \times\{1\}$ identify with $M$. The argument proceeds as follows.

Step I. 1-dimensional surgery on $M^{G}$.

Let $\alpha_{1}, \cdots, \alpha_{l}$ be a finite set of generators of $\pi_{1}\left(M^{G}\right)$. Then there exist $G$ imbeddings $f_{1}, \cdots, f_{l}$ of $S^{1} \times D^{k-1} \times D(U)$ into $M$ with disjoint images representing $\alpha_{1}, \cdots, \alpha_{l}$, respectively, where $G$-actions on $S^{1}$ and $D^{k-1}$ are trivial and $U$ is the $G$-representation on the normal fibre $\mu\left(M, M^{G}\right)_{x}$ at a point $x \in M^{G}$ in $M$ such that $U^{G}=\{0\}$. (Note that $f_{i}$ should be expressed as the $G$-imbedding of $G \times{ }_{G}\left(S^{1} \times D^{k-1} \times D(U)\right)$ into $M$. Since $G \times{ }_{G}\left(S^{1} \times D^{k-1} \times D(U)\right)$ only contains a copy of $S^{1} \times D^{k-1} \times D(U)$, we may identify $G \times{ }_{G}\left(S^{1} \times D^{k-1} \times D(U)\right)$ with $S^{1} \times D^{k-1} \times D(U)$. See $\left.[\mathrm{Br}],[\mathrm{PR}]\right)$. Let $\mathbb{S}^{1}\left(\operatorname{resp} . \mathbb{D}^{2}, \mathbb{D}^{k-1}, \mathbb{D}(U)\right)$ denote the disjoint union of $l$ copies of $S^{1}$ (resp. $\left.D^{2}, D^{k-1}, D(U)\right)$. We use the convention that $\mathbb{S}^{1} \times \mathbb{D}^{k-1} \times \mathbb{D}(U)$ (resp. $\mathbb{D}^{2} \times \mathbb{D}^{k-1} \times \mathbb{D}(U)$ ) means the disjoint union of $l$ copies of $S^{1} \times D^{k-1} \times D(U)$ (resp. $\left.D^{2} \times D^{k-1} \times D(U)\right)$.

Now, using $\mathbf{f}=\left\{f_{1}, \cdots, f_{l}\right\}$ to attach $l$ equivariant handles $\mathbb{D}^{2} \times \mathbb{D}^{k-1} \times \mathbb{D}(U)$ of type 2 to $\Gamma$ along $M \times\{1\}$ simultaneously, we obtain a new $G$-manifold

$$
\Gamma_{1}=(M \times I) \cup_{\mathbf{f}}\left(\mathbb{D}^{2} \times \mathbb{D}^{k-1} \times \mathbb{D}(U)\right) .
$$

The boundary $\partial \Gamma_{1}$ of $\Gamma_{1}$ consists of two connected components. One of them is equivariantly diffeomorphic to $M$. We denote the other one by $M_{1}$. It is easy to 
see that

$$
M_{1}=\left(M-\operatorname{Intf}\left(\mathbb{S}^{1} \times \mathbb{D}^{k-1} \times \mathbb{D}(U)\right)\right) \cup_{\mathbf{f}}\left(\mathbb{D}^{2} \times \partial\left(\mathbb{D}^{k-1} \times \mathbb{D}(U)\right)\right) .
$$

First, let us observe $\Gamma_{1}^{G}$ and $M_{1}^{G}$ in this surgery process. We see that

$$
\Gamma_{1}^{G}=\left(M^{G} \times I\right) \cup_{\left.\right|_{\mathbb{S}^{1} \times \mathbb{D}^{k-1}}}\left(\mathbb{D}^{2} \times \mathbb{D}^{k-1}\right)
$$

and

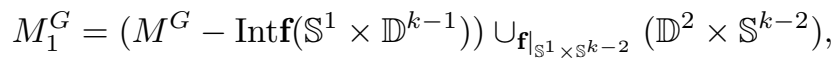

and this surgery process on $\Gamma_{1}^{G}$ and $M_{1}^{G}$ just belongs to that of the nonequivariant case. Thus from [Ke] we know that $\Gamma_{1}^{G}$ and $M_{1}^{G}$ have the following properties:

(i) $\pi_{1}\left(M_{1}^{G}\right) \cong \pi_{1}\left(\Gamma_{1}^{G}\right) \cong 0$.

(ii) $H_{i}\left(M_{1}^{G}\right) \cong 0$ if $i \neq 0,2, k-2, k ; H_{i}\left(M_{1}^{G}\right) \cong \mathbb{Z}$ if $i=0, k$. The groups $H_{2}\left(M_{1}^{G}\right)$ and $H_{k-2}\left(M_{1}^{G}\right)$ are free abelian of rank $l$.

(iii) $H_{i}\left(\Gamma_{1}^{G}\right) \cong 0$ if $i \neq 0,2, k ; H_{i}\left(\Gamma_{1}^{G}\right) \cong \mathbb{Z}$ if $i=0, k . H_{2}\left(\Gamma_{1}^{G}\right)$ is free abelian of rank $l$.

Next, we look at $\Gamma_{1}$ and $M_{1}$. It is easy to see that

$$
\pi_{1}\left(\Gamma_{1}\right) \cong \pi_{1}(\Gamma) / A
$$

and

$$
\pi_{1}\left(M_{1}\right) \cong \pi_{1}(M) / B
$$

where $A$ (resp. $B$ ) is the normal subgroup of $\pi_{1}(\Gamma)$ (resp. $\pi_{1}(M)$ ) generated by $\left.f_{i}\right|_{S^{1}}, i=1, \cdots, l$. Now we compute the homology groups of $\Gamma_{1}$ and $M_{1}$.

Claim 1. If $i \neq 0,2, n$, then $H_{i}\left(\Gamma_{1}\right) \cong 0$. $H_{0}\left(\Gamma_{1}\right) \cong H_{n}\left(\Gamma_{1}\right) \cong \mathbb{Z}$. $H_{2}\left(\Gamma_{1}\right)$ is free abelian of rank $l$.

Since $\Gamma_{1}$ has

$$
O_{1}=(M \times I) \cup_{\left.\mathbf{f}\right|_{\mathbb{S} 1}} \mathbb{D}^{2}
$$

as an $G$-deformation retract, applying the Mayer-Vietoris exact sequence of the triad $\left(O_{1} ; M \times I, \mathbb{D}^{2}\right)$, it is easy to see that Claim 1 follows.

Claim 2. If $i \neq 0,2, n-2, n$, then $H_{i}\left(M_{1}\right) \cong 0$. $H_{0}\left(M_{1}\right) \cong H_{n}\left(M_{1}\right) \cong \mathbb{Z}$. The groups $H_{2}\left(M_{1}\right)$ and $H_{n-2}\left(M_{1}\right)$ are free abelian of rank $l$.

The proof of Claim 2 can be completed by using the expression $(*)$ to compute the homology group of $M_{1}$. This is basically a standard Quillenization argument combined with a top hat construction that was much used in the 1960's. We would like to leave it as an exercise to the reader.

Step II. 2-dimensional surgery on $M_{1}^{G}$.

Let $\beta_{1}, \cdots, \beta_{l}$ and $\gamma_{1}, \cdots, \gamma_{l}$ be bases of $H_{2}\left(M_{1}^{G}\right)$ and $H_{k-2}\left(M_{1}^{G}\right)$, respectively, such that the intersection numbers $\alpha_{i} \cdot \beta_{j}=\delta_{i, j}$, where $\delta_{i, j}=1$ if $i=j$ and 0 if $i \neq j$. Then there exist disjoint $G$-imbeddings $g_{1}, \cdots, g_{l}$ of

$$
S^{2} \times D^{k-2} \times D\left(U_{1}\right)
$$

into $M_{1}$ representing the classes $\beta_{1}, \cdots, \beta_{l}$, where $G$-actions on $S^{2}$ and $D^{k-2}$ are trivial, and $U_{1}$ is the $G$-representation on the normal fibre $\mu\left(M_{1}^{G}, M_{1}\right)_{x}$ at a point $x \in M_{1}^{G}$ in $M_{1}$ such that $U_{1}^{G}=\{0\}$. Now by simultaneously attaching $l$ equivariant handles $\mathbb{D}^{3} \times \mathbb{D}^{k-2} \times \mathbb{D}\left(U_{1}\right)$ of type 3 to $\Gamma_{1}$ along $M_{1}$ via $\mathbf{g}=\left\{g_{1}, \cdots, g_{l}\right\}$, we obtain a new $G$-manifold

$$
\Gamma_{2}=\Gamma_{1} \cup_{\mathbf{g}}\left(\mathbb{D}^{3} \times \mathbb{D}^{k-2} \times \mathbb{D}\left(U_{1}\right)\right) .
$$


In two boundary components of $\Gamma_{2}$, by $M_{2}$ we denote the boundary component of $\partial \Gamma_{2}$ which is not equivariantly diffeomorphic to $M$. Then $M_{2}$ may be expressed as

$(* *) \quad M_{2}=\left(M_{1}-\operatorname{Intg}\left(\mathbb{S}^{2} \times \mathbb{D}^{k-2} \times \mathbb{D}\left(U_{1}\right)\right)\right) \cup_{\mathbf{g}}\left(\mathbb{D}^{3} \times \partial\left(\mathbb{D}^{k-2} \times \mathbb{D}\left(U_{1}\right)\right)\right)$.

Note that the above $\mathbb{D}^{3} \times \mathbb{D}^{k-2} \times \mathbb{D}\left(U_{1}\right), \mathbb{S}^{2} \times \mathbb{D}^{k-2} \times \mathbb{D}\left(U_{1}\right)$ and $\mathbb{D}^{3} \times \partial\left(\mathbb{D}^{k-2} \times \mathbb{D}\left(U_{1}\right)\right)$ fit in with the convention of Step I. The same consideration will also be used in the following discussions.

The above 2-dimensional surgery on the fixed point set is actually done in the nonequivariant case. So we know from the work of Kervaire $[\mathrm{Ke}]$ that $M_{2}^{G}$ is a homotopy sphere.

Claim 3. $\Gamma_{2}^{H}$ has the same homology as $M^{H}$ for $H=\{1\}, G$. Furthermore, $\Gamma_{2}^{G}$ is a homology cobordism between $M_{2}^{G}$ and $M^{G}$.

Since $\Gamma_{2}$ has $O_{2}=\Gamma_{1} \cup_{\left.\mathbf{g}\right|_{\mathrm{S}^{2}}} \mathbb{D}^{3}$ as its $G$-deformation retract, applying the MayerVeitoris exact sequence of the triad $\left(\mathrm{O}_{2} ; \Gamma_{1}, \mathbb{D}^{3}\right)$ and using the result (iii) and Claim 1 in Step I, it at once follows that for $H=\{1\}, G$,

$$
H_{i}\left(\Gamma_{2}^{H}\right) \cong H_{i}\left(M^{H}\right) \text {. }
$$

Next, let us observe the structure of $H_{i}\left(M_{2}\right)$. Similarly to the argument of Claim 2 , by using $(* *)$ we can still carry out the Quillenization argument, and obtain

Claim 4. $M_{2}$ is a homology sphere.

Up to now, we have finished the 1-dimensional and 2-dimensional surgeries on the fixed point set of $M$, so that the fixed point set has become a homotopy sphere. If $\pi_{1}\left(M_{2}\right)$ is trivial, then we have completed the proof. In fact, $M_{2}$ is exactly the desired semi-linear homotopy $G$-sphere $\Sigma$, and $\Gamma_{2}$ is a $G$-equivariant homology cobordism between $M$ and $\Sigma$. If $\pi_{1}\left(M_{2}\right)$ is nontrivial, we continue our discussion as follows.

Step III. Surgeries on $M_{2}-M_{2}^{G}$.

Now we begin with doing surgeries on free orbits. Since $M_{2}$ is a homology sphere, the fundamental group of $M_{2}$ is finitely presented. Let $\xi_{1}, \cdots, \xi_{t}$ be a finite set of generators for $\pi_{1}\left(M_{2}\right)$. Consider the fibration

$$
G \longrightarrow M_{2}-M_{2}^{G} \longrightarrow\left(M_{2}-M_{2}^{G}\right) / G .
$$

Since $G$ is finite, we easily see that the fundamental groups of $M_{2}-M_{2}^{G}$ and $\left(M_{2}-M_{2}^{G}\right) / G$ are isomorphic from the homotopy exact sequence of the above fibration, and there are liftings for embeddings of generators of $\pi_{1}$ of the orbit space. Thus, we may take $G$-imbeddings $h_{1}, \cdots, h_{t}$ of

$$
G \times{ }_{\{1\}}\left(S^{1} \times D^{n-1}\right)
$$

into $M_{2}$ with disjoint images representing the generators $\xi_{1}, \cdots, \xi_{t}$, where $S^{1} \times D^{n-1}$ is a $\{1\}$-space. Then, as in the arguments of Step I, we may simultaneously glue $t$ equivariant handles

$$
G \times\{1\} \mathbb{D}^{2} \times \mathbb{D}^{n-1}
$$

of type 2 to $\Gamma_{2}$ along $M_{2}$ via $\mathbf{h}=\left\{h_{1}, \cdots, h_{t}\right\}$, such that the resulting $G$-manifold denoted by $\Gamma_{3}$ has the following properties:

(1) $\Gamma_{3}^{G}=\Gamma_{2}^{G}$ and $M_{3}^{G}=M_{2}^{G}$, where $M_{3}=\partial \Gamma_{3}-(M \times\{0\})$. Note that in this surgery process, the fixed point sets $\Gamma_{3}^{G}$ and $M_{3}^{G}$ have not been touched since $\operatorname{dim} V-\operatorname{dim} V^{G} \geq 3$. 
(2) $\pi_{1}\left(M_{3}\right) \cong 0$. If $i \neq 0,2, n-2, n$, then $H_{i}\left(M_{3}\right) \cong 0 . H_{0}\left(M_{3}\right) \cong H_{n}\left(M_{3}\right) \cong \mathbb{Z}$. $H_{2}\left(M_{3}\right)$ and $H_{n-2}\left(M_{3}\right)$ are free $\mathbb{Z}[G]$-module of rank $t$.

(3) If $i \neq 0,2, n$, then $H_{i}\left(\Gamma_{3}\right) \cong 0$. $H_{0}\left(\Gamma_{3}\right) \cong H_{n}\left(\Gamma_{3}\right) \cong \mathbb{Z}$. The group $H_{2}\left(\Gamma_{3}\right)$ is free $\mathbb{Z}[G]$-module of rank $t$.

Finally, we may do 2-dimensional equivariant surgery on $\Gamma_{3}$ along $M_{3}$ to kill the basis of $H_{2}\left(M_{3}\right)$ without touching the fixed point set. It is easily verified that the resulting $G$-manifold $\Gamma_{4}$ possesses the properties that $\partial \Gamma_{4}-(M \times\{0\})$ denoted by $\Sigma$ is exactly a semi-linear homotopy $G$-sphere as desired (note that $\Sigma$ always possesses the homotopy unknottedness since the codimension $\operatorname{dim} \Sigma-\operatorname{dim} \Sigma^{G}$ is assumed to be greater than or equal to 3 ), and $\Gamma_{4}$ is a $G$-equivariant homology cobordism between $\Sigma$ and $M$. This completes the proof.

Next, we will directly use equivariant spherical modifications to prove Lemma 3.2. Since $G$ is assumed to be finite, this equivariant surgery can still be carried out well. Based upon this, we will omit the detailed computations in the proof, and only give an outline.

Proof of Lemma 3.2. Without loss of generality we assume that $W^{H}$ is non-simply connected for $H=\{1\}, G$.

We first do surgery on the fixed point set $W^{G}$ to kill the generators of $\pi_{1}\left(W^{G}\right)$. This surgery on $W^{G}$ is done in the nonequivariant case. There always exist disjoint $G$-imbeddings $\mathbf{f}=\left\{f_{1}, \ldots, f_{l}\right\}$ representing all the $l$ generators of $\pi_{1}\left(W^{G}\right)$ since $W$ is equivariantly acyclic. By doing 1-dimensional equivariant spherical modification on $W$ via $G$-imbeddings $\mathbf{f}$ to kill $\pi_{1}\left(W^{G}\right)$, we obtain a new $G$-manifold $W_{1}$ with the following properties:

(i) $\pi_{1}\left(W_{1}^{G}\right) \cong 0$. If $i \neq 0,2, k-1$, then $H_{i}\left(W_{1}^{G}\right) \cong 0$. The groups $H_{2}\left(W_{1}^{G}\right)$ and $H_{k-1}\left(W_{1}^{G}\right)$ are free $\mathbb{Z}$-module of rank $l$.

(ii) If $i \neq 0,2, n-1$, then $H_{i}\left(W_{1}\right) \cong 0$. $H_{2}\left(W_{1}\right) \cong H_{n-1}\left(W_{1}\right)$ is free $\mathbb{Z}$-module of rank $l$.

(iii) $\partial W_{1}^{H}=\partial W^{H}=M^{H}$ for $H=\{1\}, G$.

Next, we do a 2-dimensional equivariant modification to kill the basis of $H_{2}\left(W_{1}^{G}\right)$. Then the resulting $G$-manifold $W_{2}$ has the properties that $W_{2}^{G}$ becomes a contractible manifold with boundary $M^{G}$, and $W_{2}$ is still an acyclic manifold with boundary $M$.

If $W_{2}$ is not simply connected, since $G$ is finite and $\operatorname{dim} V-\operatorname{dim} V^{G} \geq 3$, then we may do 1-dimensional and 2-dimensional equivariant surgeries on the free part $W_{2}-W_{2}^{G}$ without touching the boundary and the fixed point set, creating a new contractible $G$-manifold as desired.

Now we begin with the proof of Theorem 1.3.

Proof of Theorem 1.3. Lemma 3.1 implies that $T$ is an epimorphism if $\operatorname{dim} V^{G} \geq 5$, and Lemma 3.2 implies that $T$ is a monomorphism if $\operatorname{dim} V^{G} \geq 4$ (note that for $\langle\Sigma\rangle_{G} \in \Theta_{V}^{G},\langle\Sigma\rangle_{G}=0$ if and only if $\Sigma$ bounds an equivariantly contractible $G$ manifold). Therefore, $T$ is an isomorphism if $\operatorname{dim} V^{G} \geq 5$, and a monomorphism if $\operatorname{dim} V^{G}=4$. Next, we also need to consider the low-dimensional cases, i.e., $\operatorname{dim} V^{G}=1,2$. Let $\langle M\rangle_{G} \in H \Theta_{V}^{G}$. If $\operatorname{dim} V^{G}=1$ (or 2), then $M^{G}$ is diffeomorphic to $S^{1}$ (or $S^{2}$ ). Hence, in the cases $\operatorname{dim} V^{G}=1,2$, when we need to do surgery on $M$ as an $G$-manifold, the surgery on the fixed point set $M^{G}$ need not be considered. 
Without loss of generality we assume that $M$ is nonsimply connected. We proceed as follows:

(I) The case in which either $\operatorname{dim} V^{G}=2$ or $\operatorname{dim} V^{G}=1$ and $\operatorname{dim} V \geq 5$. In this case, we claim that $T$ is an isomorphism. Since $\operatorname{dim} V \geq 5$, we may change $M$ into a semi-linear homotopy $G$-sphere $\Sigma$ by doing surgeries without touching the fixed point set, such that $M$ is $G$-equivariantly homologically cobordant to $\Sigma$, i.e., $\Sigma$ may be used as a representative of the class $\langle M\rangle_{G}$. This means that if either $\operatorname{dim} V^{G}=2$ or $\operatorname{dim} V^{G}=1$ and $\operatorname{dim} V \geq 5$, then $T$ is an epimorphism. If $\langle M\rangle_{G}=0$, i.e., $M$ bounds an equivariantly acyclic $G$-manifold $W$, then in a same way as the proof of Lemma 3.2, it is easy to check that $M$ bounds an equivariantly contractible $G$-manifold. This means that if either $\operatorname{dim} V^{G}=2$ or $\operatorname{dim} V^{G}=1$ and $\operatorname{dim} V \geq 5$, then $T$ is a monomorphism.

(II) The case in which $\operatorname{dim} V^{G}=1$ and $\operatorname{dim} V=4$. In this case, we may directly compute the groups $H \Theta_{V}^{G}$ and $\Theta_{V}^{G}$. By [Ke, Theorem 3], we know that every 4-dimensional homology sphere bounds a contractible manifold. Thus if $\operatorname{dim} V^{G}=1$ and $\operatorname{dim} V=4$, then the semi-linear homology $G$-sphere $M$ bounds an equivariantly contractible $G$-manifold. This immediately deduces that

$$
H \Theta_{V}^{G} \cong \Theta_{V}^{G} \cong 0
$$

if $\operatorname{dim} V^{G}=1$ and $\operatorname{dim} V=4$.

Combining the above discussions, we complete the proof.

Remark. (1) If $\operatorname{dim} V^{G}=4$, the above argument tells us that $T$ is nothing but a monomorphism. Let $\operatorname{dim} V^{G}=4$ and let the $G$-action be semi-free. Consider the homomorphism

$$
K_{1}: H \Theta_{V}^{G} \longrightarrow H \Theta_{\operatorname{dim} V}
$$

defined by mapping $\langle M\rangle_{G} \in H \Theta_{V}^{G}$ to $\langle M\rangle \in H \Theta_{\operatorname{dim} V}$ by forgetting the $G$-action. If

$$
K_{1}\left(\langle M\rangle_{G}\right)=\langle M\rangle=0
$$

in $H \Theta_{\operatorname{dim} V}$, then $M$ is homologically cobordant to $S^{\operatorname{dim} V}$ so $M$ bounds an acyclic manifold. By [Ke, Theorem 3], every 4-dimensional homology sphere always bounds an acyclic manifold, thus $M^{G}$ as a homology 4-sphere bounds an acyclic manifold. Since the $G$-action is assumed to be semi-free, we conclude that $M$ equivariantly bounds an equivariant acyclic $G$-manifold. Furthermore, by Lemma 2.1 it follows that $M$ is $G$-equivariantly homologically cobordant to $S(V \oplus \mathbb{R})$, i.e., $\langle M\rangle_{G}=$ 0 . Therefore, $K_{1}$ is a monomorphism. Since $H \Theta_{\operatorname{dim} V}$ is finite, $H \Theta_{V}^{G}$ is finite if $\operatorname{dim} V^{G}=4$. In a same way, we may also define the homomorphism

$$
K_{2}: \Theta_{V}^{G} \longrightarrow \Theta_{\operatorname{dim} V}
$$

by forgetting the $G$-action, and obtain that $K_{2}$ is a monomorphism too, so $\Theta_{V}^{G}$ is finite if $\operatorname{dim} V^{G}=4$. Obviously, if either $K_{1}$ and $K_{2}$ are epimorphisms or $\operatorname{Im} K_{1}$ and $\operatorname{Im} K_{2}$ are isomorphic, then we can conclude that $T$ is an isomorphism if $\operatorname{dim} V^{G}=4$. However, we do not know whether each homology (resp. homotopy) sphere of dimension $\geq 7$ must admit a semi-free $G$-action such that the fixed point 
set is a homology (resp. homotopy) 4-sphere. Even so, it is extremely tempting to conjecture that $T: \Theta_{V}^{G} \rightarrow H \Theta_{V}^{G}$ is an isomorphism if $\operatorname{dim} V^{G}=4$.

(2) If $G$-actions are not assumed to be semi-free and $\operatorname{dim} V^{G}$ is not restricted to be 4 , and $G$ is not necessarily finite, by forgetting $G$-actions we can still obtain two homomorphisms like $K_{1}$ and $K_{2}$, so that the following commutative diagram can be obtained:

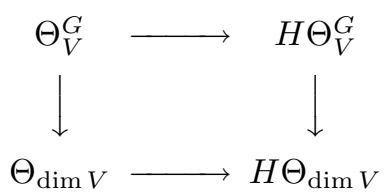

This gives a connection between ordinary cases and equivariant cases.

\section{ACKNOWLEDGMENTS}

The author expresses his gratitude to Professors T. Tsuboi and M. Masuda for their advice and suggestions in this work. The author also expresses his gratitude to the referee, who did an extremely careful reading of this paper and detected some flaws in the proof of the main result in earlier versions. The many suggestions and comments made by him considerably improved the presentation of this paper, leading to the present final version.

\section{REFERENCES}

[AB] M. F. Atiyah and R. Bott, A Lefschetz fixed point formula for elliptic complexs. II: Applications, Ann. of Math. (2) 88 (1968), 451-491. MR 38:731

[Br] G. E. Bredon, Introduction to compact transformation groups, Academic Press, 1972. MR 54:1265

[FS] R. Fintushel and R. Stern, Pseudofree orbifolds, Ann. of Math. 122 (1985), 335-364. MR 87a:57027

[Fu] M. Furuta, Homology cobordism group of homology 3-spheres, Invent. Math. 100 (1990), 339-355. MR 91c:57039

$[\mathrm{HH}]$ W. C. Hsiang and W. Y. Hsiang, Differentiable actions of compact connected classical groups I, Amer. J. Math. 89 (1967), 705-786. MR 36:304

[Ke] M. Kervaire, Smooth homology spheres and their fundamental groups, Trans. Amer. Math. Soc. 144 (1969), 67-72. MR 40:6562

[KM] M. Kervaire and J. Milnor, Groups of homotopy spheres, Ann. of Math. 77 (1963), 504537. MR 26:5584

[Ko] A. Kosiński, On the inertia groups of $\pi$-manifolds, Amer. J. Math. 89 (1967), 227-248. MR 35:4936

[Ma] M. Masuda, A product formula for connected sum, Transformation Groups (Proceedings, Osaka, 1987), Lecture Notes in Math. Vol. 1375, Springer, Berlin-Heidelberg-New YorkTokyo, 1989, 231-239. MR 90k:57044

[MSc1] M. Masuda and R. Schultz, Invariants of Atiyah-Singer type, classifications up to finite ambiguity, and equivariant inertia groups, Indiana Univ. Math. J. 45 (1996), 545-581. MR 97i:57032

[MSc2] M. Masuda and R. Schultz, On the nonuniquences of equivariant connected sums, J. Math. Soc. Japan 51 (1999), 411-435. MR 2000b:57049

[Mi1] J. Milnor, Lecture on the h-cobordism theorem, Princeton University Press, 1965. MR 32:8352

[Mi2] J. Milnor, Whitehead torsion, Bull. Amer. Math. Soc. 72 (1966), 358-426. MR 33:4922

[PR] T. Petrie and J. D. Randall, Transformation groups on manifolds, Monographs and textbooks in pure and applied mathematics, 1984. MR 85m:57026

[Sc1] R. Schultz, On the inertia group of a product of spheres, Trans. Amer. Math. Soc. 156 (1971), 137-153. MR 43:1209 
[Sc2] R. Schultz, Differentiable group actions on homotopy spheres II: Ultrasemi-free actions, Trans. Amer. Math. Soc. 268 (1981), 255-297. MR 83a:57055

[Sc3] R. Schultz, Nonlinear analogs of linear group actions on spheres, Bull. Amer. Math. Soc. (2) 11 (1984), 263-285. MR 86i:57049

[Wi1] D. L. Wilkens, On the inertia groups of certain manifolds, J. London Math. Soc. (2) 9 (1975), 537-548. MR 52:4316

[Wi2] D. L. Wilkens, On inertia groups and bordism, Michigan Math. J. 23 (1976), 105-106. MR 53:14511]

[Win] H. E. Winkelnkemper, On the action of $\Theta^{n}$, Trans. Amer. Math. Soc. 206 (1975), 339-346. MR 54:1257

Institute of Mathematics, Fudan University, Shanghai, 200433, People's Republic of CHINA

E-mail address: zlu@fudan.edu.cn

Current address: Department of Mathematics, Osaka City University, 3-3-138 Sugimoto, Sumiyoshi-ku, Osaka 558-8585, Japan 